\title{
Specificity vs versatility: A fine balance for novel targeted molecular imaging radiotracers
}

\author{
James T. Thackeray, PhD, ${ }^{a}$ and Frank M. Bengel, $M^{a}$ \\ a Department of Nuclear Medicine, Hannover Medical School, Hannover, Germany
}

Received Jan 21, 2016; accepted Jan 21, 2016

doi:10.1007/s12350-016-0426-2

\section{See related article, pp. 558-570}

The inherent strength of nuclear cardiology imaging lies in its high sensitivity and capacity for dynamic longitudinal interrogation of evolving (patho)physiologic processes. The ability to monitor molecular changes in the development and resolution of disease is a powerful tool for clinical research and practice.

${ }^{18} \mathrm{~F}$-Deoxyglucose $\left({ }^{18} \mathrm{~F}-\mathrm{FDG}\right)$ has been the vanguard of molecular imaging, finding a niche not only in cardiac metabolism, but also as a marker of inflammation due to uptake by activated inflammatory cells. ${ }^{1}$ Taken in perspective, the versatility of ${ }^{18} \mathrm{~F}$-FDG is quite remarkable. But this target flexibility can also limit the effectiveness of FDG for molecular imaging. In recent years, a widening array of novel or repurposed tracers targeted to specific physiologic mechanisms, cell types, or receptors have emerged to evaluate various components of the disease process. To this end, recent studies have evaluated novel tracers targeting chemokine receptors on leukocytes (e.g., ${ }^{68}$ Ga-pentixafor), ${ }^{2}$ somatostatin receptors on activated macrophages (e.g., ${ }^{68}$ Ga-DOTATATE), ${ }^{3}$ mannose receptor on M2-polarized macrophages (e.g., ${ }^{18} \mathrm{~F}$-mannose), ${ }^{4} \alpha \mathrm{v} \beta 3$ integrin expression in atherosclerotic plaque (e.g., ${ }^{18} \mathrm{~F}$-galacto-RGD), ${ }^{5}$ elastin expression by vascular endothelial cells (e.g., ${ }^{64} \mathrm{Cu}-\mathrm{G} 1 \mathrm{C}$ glycoprotein), ${ }^{6}$ or matrix metalloproteinase expression in extracellular matrix (e.g., $\left.{ }^{18} \mathrm{~F}-\mathrm{RP} 802\right) .{ }^{7}$

Another emerging biomarker is sphingosine-1 phosphate (S1P), a soluble chemoattractant cytokine involved in postnatal vascular development, immune cell homing, endothelial cell permeability, and vascular

Reprint requests: James T. Thackeray, PhD, Department of Nuclear Medicinem, Hannover Medical School, Carl-Neuberg-Str. 1, 30625

Hannover, Germany; thackeray.james@mh-hannover.de

J Nucl Cardiol 2017;24:571-3.

1071-3581/\$34.00

Copyright (c) 2016 American Society of Nuclear Cardiology. smooth muscle migration. In the current issue of the Journal, Jin and colleagues present a preliminary evaluation of the novel sphingosine-1-phosphate receptor 1 $\left(\mathrm{S}_{1} \mathrm{P}_{1}\right)$ ligand ${ }^{11} \mathrm{C}$-TZ3321 for molecular PET imaging of vascular injury, specifically targeting neointimal hyperplasia. ${ }^{8}$ Expansion of the vascular intima is associated with increased $\mathrm{S}_{1} \mathrm{P}_{1}$ expression by invading smooth muscle cells, providing an enticing imaging target to non-invasively assess vascular remodeling. In a clinical setting, $\mathrm{S}_{1} \mathrm{P}_{1}$-expressing vascular smooth muscle cells contribute to in-stent restenosis, a situation for which an imaging biomarker may be of significant benefit.

Jin and colleagues report that ${ }^{11} \mathrm{C}$-TZ3321 exhibits high affinity and selectivity for $\mathrm{S}_{1} \mathrm{P}_{1}$ in vitro, and displays favorable biodistribution in healthy animals, though basal tracer accumulation in non-target organs is relatively high. Small animal PET imaging after femoral artery denudation injury demonstrates a sustained increase in ${ }^{11} \mathrm{C}$-TZ3321 binding localized to the injured artery of $\mathrm{ApoE}^{-1-}$ mice compared to both the contralateral leg and to wild-type mice over 4 weeks after arterial injury. The elevated binding was associated with increased intimal thickness, vascular smooth muscle content, and positive $\mathrm{S}_{1} \mathrm{P}_{1}$ immunostaining. By contrast, ${ }^{18}$ F-FDG uptake at 1 week post-injury did not differ between strains and was localized superficial to the injured artery, suggesting non-specific inflammation related to surgical procedure. Taken together, these findings suggest that ${ }^{11} \mathrm{C}$-TZ3321 can distinguish vascular smooth muscle expansion and intimal thickening in a manner distinct from ${ }^{18} \mathrm{~F}-\mathrm{FDG}$.

$\mathrm{S} 1 \mathrm{P}$ receptors are an interesting imaging target considering their complex signaling, regulation, and expression patterns. Physiologically, S1P receptors mediate vascular permeability, which may suggest a wider application in terms of a surrogate marker of inflammation, early in the cell infiltration process. Moreover, S1P is implicated in the beneficial effects of high density lipoproteins (HDL), and non-invasive 
evaluation of receptor expression may provide some insight into the complex role of HDL. It is important to note that the retention patterns of ${ }^{11} \mathrm{C}-\mathrm{TZ} 3321$ and ${ }^{18} \mathrm{~F}$ FDG are distinct, reflecting different imaging targets and potentially different stages in disease progression, a microcosm of the oppositional forces of specificity and versatility.

Moreover, different subtypes of S1P receptors mediate different pathways. For example, $\mathrm{S}_{1} \mathrm{P}_{2}$ is thought to promote inflammation, whereas $\mathrm{S}_{1} \mathrm{P}_{1}$ is thought to suppress it. ${ }^{9,10}$ In the case of vascular smooth muscle cells in neointimal hyperplasia, $\mathrm{S}_{1} \mathrm{P}_{1}$ is an attractive and likely selective target, whereas $\mathrm{S}_{1} \mathrm{P}_{2}$ or $\mathrm{S}_{1} \mathrm{P}_{3}$ binding may be preferred in other conditions. Acute balloon injury in rat carotid artery evokes an early increase in $\mathrm{S}_{1} \mathrm{P}_{1}$ mRNA to 72 hours, followed by a smaller increase in $\mathrm{S}_{1} \mathrm{P}_{2}$ mRNA at $\sim 10$ days. ${ }^{11}$ Such complex regulation poses the question: does the targeting of one S1P receptor subtype focus or blur the true physiology of the disease progression? In the case of imaging vascular smooth muscle cells, tracer specificity is clearly beneficial, but does this impact the wider application of the tracer? An ancillary benefit may be found in the parallel development of S1P-targeted pharmaceuticals such as FTY720, which provides the direct opportunity for monitoring of therapeutic effect. Indeed, a recent report has detailed the labeling of a series of FTY720 analogs with fluorine-18 for PET imaging. ${ }^{12}$ It should be noted that as a carbon-11-labeled tracer, ${ }^{11} \mathrm{C}$-TZ3321 production requires an on-site cyclotron; further rollout necessitates the development of an ${ }^{18} \mathrm{~F}$-labeled analog.

Jin and colleagues have provided a thorough and wellcontrolled preliminary evaluation of ${ }^{11} \mathrm{C}-\mathrm{TZ} 3321$ as a lead compound for PET imaging of $\mathrm{S}_{1} \mathrm{P}_{1}$. Their study is not without some limitations. Firstly, the wire-induced femoral artery denudation model has important differences from clinical manifestation of in-stent restenosis. In particular, while denudation of a native artery promotes intimal thickening and vascular smooth muscle cell proliferation, the signaling factors (including S1P) cannot directly mimic the coronary stent microenvironment in vivo. It is curious that the milder intimal thickening in wild-type mice does not evoke a clear $\mathrm{S}_{1} \mathrm{P}_{1} \mathrm{TZ} 3321$ signal. While the authors accurately point out that the majority of patients with stents have a history of hyperlipidemia, the severity is exaggerated in the $\mathrm{ApoE}^{-1-}$ mouse.

Secondly, the findings by Jin and colleagues are largely observational and tracer-dependent. While they have reported on the increased intimal thickness in the wire-injured femoral arteries of $\mathrm{ApoE}^{-/-}$mice compared to those of wild-type mice, there is no direct quantification of $\mathrm{S}_{1} \mathrm{P}_{1}$ beyond qualitative immunostaining. The timecourse of tracer distribution is somewhat problematic, in that the signal in the injured artery does not evolve over time, remaining constant over 4 weeks after wire injury, whereas neointimal thickening in the model has been described as a progressive process. ${ }^{13}$ In the absence of serial histopathology, it is difficult to reconcile the imaging information with the severity of the vascular smooth muscle cell proliferation and neointimal hyperplasia. Clearly, ${ }^{11} \mathrm{C}$-TZ3321 shows a higher sensitivity to these changes than ${ }^{18} \mathrm{~F}-\mathrm{FDG}$, but the prognostic power and clinical relevance will need to be established. Additional indirect evidence supports the specificity of ${ }^{11} \mathrm{C}$-TZ3321 for vascular smooth muscle cells in this setting, but the precise cells responsible for the in vivo image are not directly identified - the resolution of autoradiography, let alone PET, is insufficient to resolve the cellular or regional binding due to the very small size of the femoral artery. Flow cytometry may allow a more robust cellular characterization of in vivo ${ }^{11} \mathrm{C}$-TZ3321 uptake.

Thirdly, the feasibility to translate findings from a fairly remote peripheral artery, distant from the high lung, liver and heart activity (3-60\% ID/g at 60 minutes post-injection, per biodistribution) remains unclear. Indeed, the in vivo images presented in the manuscript illustrate intense activity in the abdominal and thoracic cavity, which would interfere with imaging arteries located in these regions. Certainly, tracer clearance can differ widely between mice and humans, ${ }^{3,14}$ but acceptably low background will be of critical importance for the intended target of in-stent restenosis.

Finally, the comparison of ${ }^{11} \mathrm{C}$-TZ3321 to ${ }^{18} \mathrm{~F}$-FDG is limited to a single, early timepoint (1 week) in the disease progression. While the tracer signals appear to reflect different pathological processes (vascular smooth muscle cell proliferation vs post-surgical inflammation, respectively), it would be interesting to know whether ${ }^{18}$ F-FDG uptake changes over the timecourse of the intimal thickening.

These limitations notwithstanding, the work contributes to a growing body of literature on molecular imaging in nuclear cardiology. As more specific molecular targets of disease are identified, the balance between developmental effort and clinical payoff must be negotiated. Highly specific tracers may provide excellent image clarity, but does the research and development effort justify the perceived advantage over ${ }^{18}$ F-FDG? The demonstration of such benefit relies on a combination of prognostic value and the evaluation of therapy. Outcome-based strategies are paramount-the ability to detect a difference in tracer binding between health and disease states is only the first step; rather, it is essential that a candidate tracer determines not only the presence of abnormalities, but also defines the prognostic value or evaluate the resolution of these 
abnormalities by therapeutic intervention., ${ }^{2,15}$ It appears that ${ }^{11} \mathrm{C}$-TZ3321 can distinguish between mild (wild-type) and more severe $\left(\mathrm{ApoE}^{-/-}\right)$neointimal hyperplasia (albeit with limited binding in the mild phenotype), but it will be important to determine whether the tracer has sufficient sensitivity to identify mild vascular smooth muscle proliferation before overt disease. The molecular targeting of the tracer underlies the potential to exceed identification of restenosis by anatomical imaging alone. Highly controlled serial imaging and correlation to physiologically significant stenosis will be important to empirically demonstrate such added value.

Moreover, recent studies have established the power of molecular imaging to evaluate the resolution of disease and response to therapeutic intervention. For example, the CXCR4-specific ${ }^{68} \mathrm{Ga}$-pentixafor in vivo PET signal was responsive to anti-inflammatory therapy in mice after myocardial infarction, ${ }^{2}$ and ${ }^{18} \mathrm{~F}$-galactoRGD uptake in atherosclerotic plaques declined after cessation of atherogenic diet in mice. ${ }^{16}$ Pharmacological blockade of ${\mathrm{S} 1 \mathrm{P}_{1}}_{1}$ by FTY720 after femoral artery denudation is a logical next step, and may provide valuable insight into the sensitivity of the tracer and its suitability for monitoring therapeutic efficacy. Angiotensin receptor blockade has also been suggested to inhibit neointimal hyperplasia, ${ }^{17}$ providing an alternative approach for comparison of non-targeted, clinically relevant therapy.

Expanding the clinical repertoire for molecular imaging beyond ${ }^{18} \mathrm{~F}-\mathrm{FDG}$ is an arduous undertaking. Regulation will remain a fundamental obstacle for translating preclinical findings to clinical practice. Nonetheless, novel tracers with an established application can provide important complementary information for diagnosis and prognosis of cardiovascular disease. Studies such as Jin and colleagues are critical to the continued evolution of molecular imaging, striving to attain the fine balance between specificity and versatility.

\section{Disclosure}

The authors declare that they have no conflict of interest.

\section{References}

1. Lee WW, Marinelli B, van der Laan AM, Sena BF, Gorbatov R, Leuschner $\mathrm{F}$, et al. PET/MRI of inflammation in myocardial infarction. J Am Coll Cardiol 2012;59:153-63.

2. Thackeray JT, Derlin T, Haghikia A, Napp LC, Wang Y, Ross TL, et al. Molecular imaging of the chemokine receptor CXCR4 after acute myocardial infarction. JACC Cardiovasc Imaging 2015;8: 1417-26.

3. Schatka I, Wollenweber T, Haense C, Brunz F, Gratz KF, Bengel FM. Peptide receptor targeted radionuclide therapy alters inflammation in atherosclerotic plaques. J Am Coll Cardiol 2013;62: 2344-5.

4. Tahara N, Mukherjee J, de Haas HJ, Petrov AD, Tawakol A, Haider $\mathrm{N}$, et al. 2-Deoxy-2-[18F]fluoro-D-mannose positron emission tomography imaging in atherosclerosis. Nat Med 2014;20:215-9.

5. Laitinen I, Saraste A, Weidl E, Poethko T, Weber AW, Nekolla $\mathrm{SG}$, et al. Evaluation of alphavbeta3 integrin-targeted positron emission tomography tracer 18F-galacto-RGD for imaging of vascular inflammation in atherosclerotic mice. Circ Cardiovasc Imaging 2009;2:331-8.

6. Bigalke B, Phinikaridou A, Andia ME, Cooper MS, Schuster A, Schonberger $\mathrm{T}$, et al. Positron emission tomography/computed tomographic and magnetic resonance imaging in a murine model of progressive atherosclerosis using (64)Cu-labeled glycoprotein VI-Fc. Circ Cardiovasc Imaging 2013;6:957-64.

7. Golestani R, Razavian M, Nie L, Zhang J, Jung JJ, Ye Y, et al. Imaging vessel wall biology to predict outcome in abdominal aortic aneurysm. Circ Cardiovasc Imaging 2015;8:e02471.

8. Jin H, Yang H, Liu H, Zhang Y, Zhang X, Rosenberg AJ, et al. A promising carbon-11-labeled sphingosine-1-phosphate receptor 1specific PET tracer for imaging vascular injury. J Nucl Cardiol 2016. doi:10.1007/s12350-015-0391-1.

9. Blaho VA, Hla T. An update on the biology of sphingosine 1phosphate receptors. J Lipid Res 2014;55:1596-608.

10. Swan DJ, Kirby JA, Ali S. Vascular biology: The role of sphingosine 1-phosphate in both the resting state and inflammation. $\mathbf{J}$ Cell Mol Med 2010;14:2211-22.

11. Wamhoff BR, Lynch KR, Macdonald TL, Owens GK. Sphingosine-1-phosphate receptor subtypes differentially regulate smooth muscle cell phenotype. Arterioscler Thromb Vasc Biol 2008;28:1454-61.

12. Schilson SS, Keul P, Shaikh RS, Schafers M, Levkau B, Haufe G. Synthesis of new ligands for targeting the S1P1 receptor. Bioorg Med Chem 2015;23:1011-26.

13. Roque M, Fallon JT, Badimon JJ, Zhang WX, Taubman MB, Reis ED. Mouse model of femoral artery denudation injury associated with the rapid accumulation of adhesion molecules on the luminal surface and recruitment of neutrophils. Arterioscler Thromb Vasc Biol 2000;20:335-42.

14. Thackeray JT, Bankstahl JP, Wang Y, Korf-Klingebiel M, Walte A, Wittneben A, et al. Targeting post-infarct inflammation by PET imaging: Comparison of (68)Ga-citrate and (68)Ga-DOTATATE with (18)F-FDG in a mouse model. Eur J Nucl Med Mol Imaging 2015;42:317-27.

15. Razavian M, Tavakoli S, Zhang J, Nie L, Dobrucki LW, Sinusas $\mathrm{AJ}$, et al. Atherosclerosis plaque heterogeneity and response to therapy detected by in vivo molecular imaging of matrix metalloproteinase activation. J Nucl Med 2011;52:1795-802.

16. Saraste A, Laitinen I, Weidl E, Wildgruber M, Weber AW, Nekolla SG, et al. Diet intervention reduces uptake of alphavbeta3 integrin-targeted PET tracer 18F-galacto-RGD in mouse atherosclerotic plaques. J Nucl Cardiol 2012;19:775-84.

17. Yamada T, Kondo T, Numaguchi Y, Tsuzuki M, Matsubara T, Manabe I, et al. Angiotensin II receptor blocker inhibits neointimal hyperplasia through regulation of smooth muscle-like progenitor cells. Arterioscler Thromb Vasc Biol 2007;27:2363-9. 